\title{
A Study on Flow Patterns of Oscillatory Thermocapillary Convection in Floating Half Zone by PIV
}

\author{
Y. Aa, Z. H. Cao, W. R. Hu \\ National Microgravity Laboratory / CAS, Institute of Mechanics, \\ Chinese Academy of Sciences, Beijing 100080, China \\ E-mail:ay@imech.ac.cn
}

Corresponding author Y. Aa

\begin{abstract}
The velocity fields of oscillatory convection have been measured using the techniques of Particle Image Velocimetry (PIV) in a liquid bridge of half floating zone with small typical scales of a few millimeters for emphasizing the thermocapillary effect in comparison with the effect of buoyancy. The flow patterns of the oscillatory flow have been studied experimentally in a liquid bridge. The flow patterns in the liquid bridge are classified with mode numbers according to oscillatory flow characteristics. Results of the experiment show that the mode depends on the aspect ratio as well as the volume ratio of the liquid bridge. The experimental results are helpful for studying the structure of flow at the onset of oscillatory thermocapillary convection in a liquid bridge.
\end{abstract}

Key words: thermocapillary convection, PIV, liquid bridge

\section{Introduction}

Fluid convection, which is driven by temperature gradient along its free surface, is called thermocapillary convection. Under a microgravity environment among many possible convection phenomena, the thermocapillary convection is generally dominant compared to the buoyancy convection in a fluid system with free surface. Therefore thermocapillary convection is of special importance to Microgravity Science and Engineering. In space crystal growth processes, like the floating-zone method, surface tension driven convection can be very important to the final quality of crystals. Ostrach et all [1] presented the dynamic Bond number ( $B d=\rho g \beta d 2 / \sigma T)$ in order to simulate a microgravity environment for a floating zone configuration in a ground based work, and suggested that the Bond number be less than unity. In general, the characteristic length $\mathrm{d} 0$ of the floating zone must be kept small, for example several $\mathrm{mm}$ in height. A small-size half-floating zone device, called the liquid bridge, is usually used to study thermocapillary convection on the ground for simulating a space microgravity environment.

In the present paper, a Particle Image Velocimetry is successfully used to quantitatively measure the fluid velocity field in the horizontal cross-section of a liquid bridge in real time. The different flow patterns of the oscillatory flows have been studied experimentally and have been classified with mode numbers according to the oscillatory flow characteristics.

Y. Aa, Z. H. Cao, W. R. Hu

National Microgravity Laboratory / CAS, Institute of Mechanics

Beijing China 


\section{Experimental Facility}

Our experimental facility (see Fig. 1) is a special system of floating half zone, which is different from a usual model of floating half zone consisting of a liquid bridge persisted between two copper rods. Our liquid bridge of $10 \mathrm{cst}$ silicone oil (see Table 1 for the fluid properties) is floated in the gap between a lower copper rod and a transparent upper disk consisting of $\mathrm{K} 9$ glass of $4 \mathrm{~mm}$ in diameter, with an upper disk used for visual measurement. The upper disk of glass is surrounded by a heater with copper ring. The lower rod of copper and upper disk of glass surrounded by a ring heater are co-axial, and are of same diameter of $d_{0}=5 \mathrm{~mm}$.

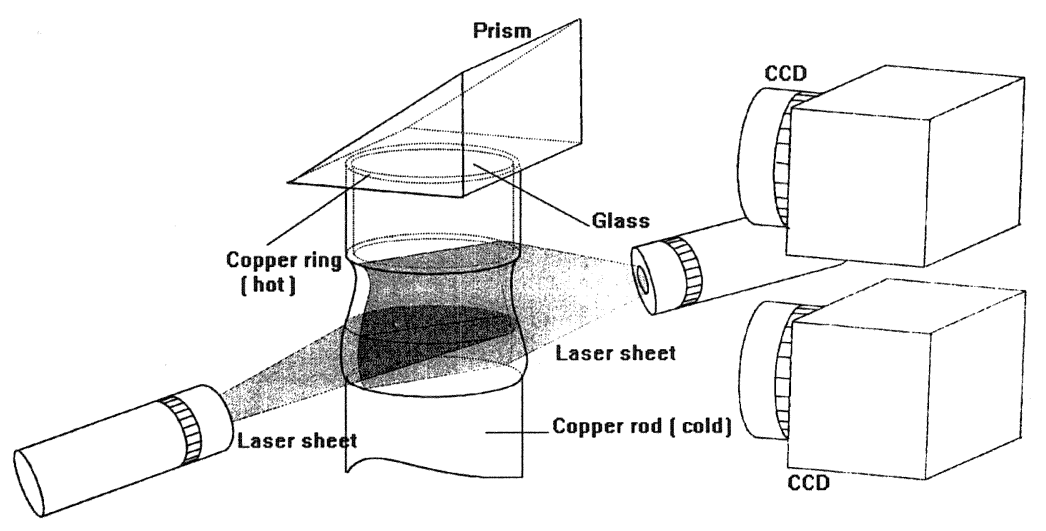

Fig.1. Schematic diagram of experimental facility

Table 1. Fluid properties of silicone oil.

\begin{tabular}{|ccccccccc|}
\hline Fluid & $\sigma$ & $\sigma^{\prime}{ }_{\mathrm{T}}$ & $\rho$ & $\mathrm{v}$ & $\mathrm{K}$ & $\mathrm{K}$ & $\mathrm{P}_{\mathrm{r}}$ & $\beta$ \\
& $\mathrm{kg} / \mathrm{s}^{2}$ & $\mathrm{~kg} / \mathrm{s}^{2} \cdot{ }^{\circ} \mathrm{C}$ & $\mathrm{kg} / \mathrm{m}^{3}$ & $\mathrm{~m}^{2} / \mathrm{s}$ & $\mathrm{Cal} / \mathrm{ms} \cdot{ }^{\circ} \mathrm{C}$ & $\mathrm{m}^{2} / \mathrm{s}$ & & $1 /{ }^{\circ} \mathrm{C}$ \\
& & & & & & & & \\
silicone oil & & & & & & & \\
10cst & 0.0201 & $-0.6 \times 10^{-4}$ & $0.935 \times 10^{3}$ & $1 \times 10^{-5}$ & $3.7 \times 10^{-5}$ & $9.47 \times 10^{-8}$ & 105.6 & $1.08 \times 10^{-3}$ \\
\hline \hline
\end{tabular}

The temperature distribution in the upper glass disk is estimated to be nearly constant in radial direction due to the larger heat conductivity of the $\mathrm{K} 9$ glass in comparison with the one of 10cst silicone oils [2]. This means that the model of the present paper is close to the usual model of floating half zone with constant temperatures at both copper rods.

A system of digital particle image velocimetry (DPIV) of DANTEC measurement technology A/S with Flow Map PIV 2000 Processor was used to quantitatively measure the 2D velocity field in a horizontal cross-section of the liquid bridge, as shown in Fig.2. The sequence of velocity fields in one period of flow oscillations was emphasized.

Hollow glass spheres $10 \mu \mathrm{m}$ in diameter as tracers were suspended in the liquid bridge. The density of the hollow glass spheres is $1.1 \mathrm{~g} / \mathrm{cm}^{3}$, which is close to the density of 10cst silicone oil (0.93 $\left.\mathrm{g} / \mathrm{cm}^{3}\right)$. 


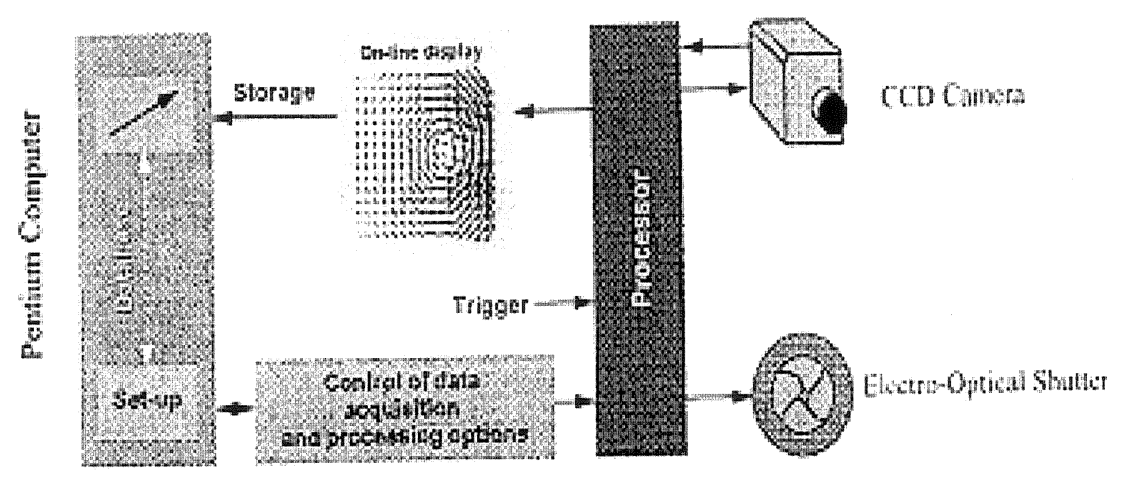

Fig.2. Schematic diagram of DPIV system

An argon ion laser of $1 \mathrm{~W}$ in power was applied to illuminate the flow field. Pulse lights were shaped by an electro-optical shutter, and controlled by a center processor with CCD camera synchronization. The resolution of the CCD camera is $768 \times 484$ pixels (with $11.6 \times 13.6 \mu \mathrm{m}$ pixel pitch). Because the flow field of the small liquid bridge is only $4 \mathrm{~mm}$ in diameter, it was enlarged to about 2 times on the CCD plane. Using a cylindrical lens, a $1.0 \mathrm{~mm}$ thin light sheet was shaped to illuminate a horizontal cross-section of the liquid bridge. The acquisition technique for flow field is based on the record of particle reflection in the liquid. The cross-correlation technique of DPIV image matching processing was used to obtain both the magnitude and direction of velocity vector at the same time [3].

In order to ensure the accuracy of our measurements, we calibrated the velocity of particles using a 2 -D micropositioning system. Results show that the velocity measurement accuracy is greater than $5 \%$.

\section{Experimental results}

Thermocapillary convection in the liquid bridge is caused by a surface tension gradient, since there is temperature distribution on the free surface of the liquid bridge. The applied temperature difference $\Delta \mathrm{T}$ between the upper heater of copper ring and the cold lower rod is gradually increased during the experimental procedure. With the increase of $\Delta \mathrm{T}$, convection transits from steady into oscillation. The flow pattern of steady convection is axis-symmetric in the vertical section, and azimuthal velocity is zero in the horizontal cross-section. When $\Delta \mathrm{T}$ is increased beyond a certain critical value $(\Delta \mathrm{T})_{\mathrm{c}}$ the flow pattern transits from axisymmetric to asymmetric. Fig.3 and Fig.4 give a sequence of flow patterns of a vertical cross-section and a horizontal cross-section in one oscillatory period. Rotating dark region associates with low speed flow with fewer tracers, but does not mean the rotation of medium particles. Because of the oscillation period in the present experimental conditions is 1.49 second, a time interval between frames is 0.37 second corresponding to a quarter of one period. Fig.5 give a two-dimensional velocity field in a horizontal cross-section in one oscillatory period in condition same as Fig.4.

In the liquid bridges of the different aspect ratio, we obtained fluid velocity fields of the horizontal sections; see the Fig. 6 . When volume ratio of liquid bridge $V / \mathrm{V}_{0}=1$,

\begin{tabular}{|c|c|c|}
\hline $\begin{array}{l}\text { Aspect Ratio } \\
l / d_{0}\end{array}$ & $\begin{array}{l}\text { Azimuthal Mode } \\
\mathrm{m}\end{array}$ & Related State \\
\hline 0.8 & 1 & oscillatory \\
\hline 0.6 & 2 & oscillatory \\
\hline 0.4 & 3 & oscillatory \\
\hline
\end{tabular}




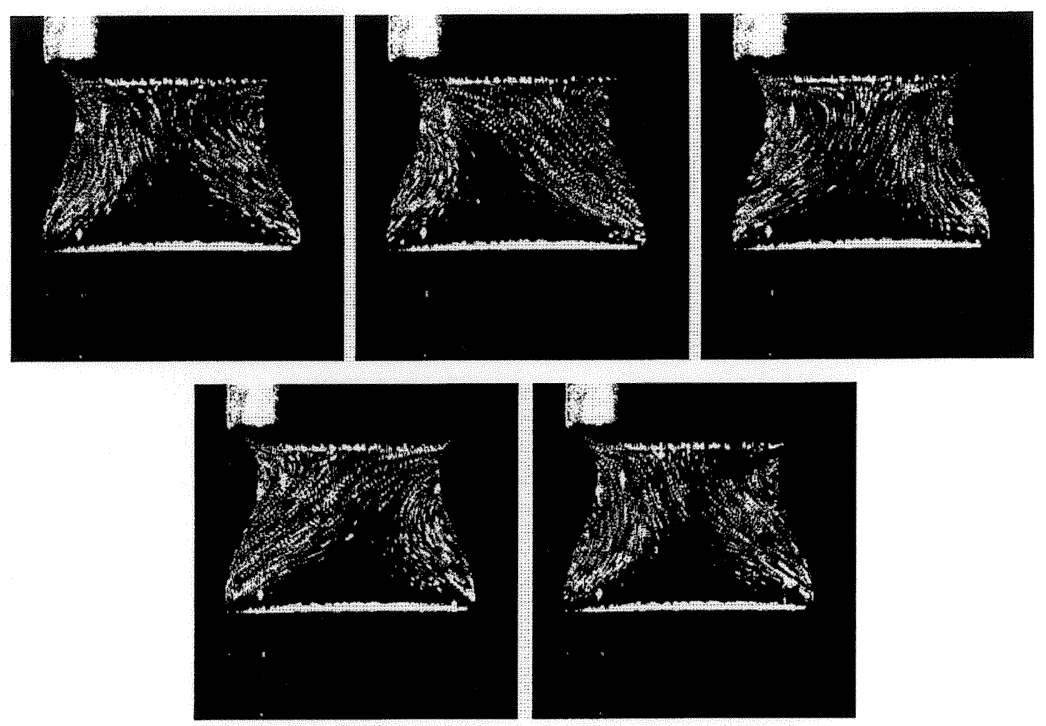

Fig.3. A sequence of flow pattern in the vertical cross-section in one oscillatory period

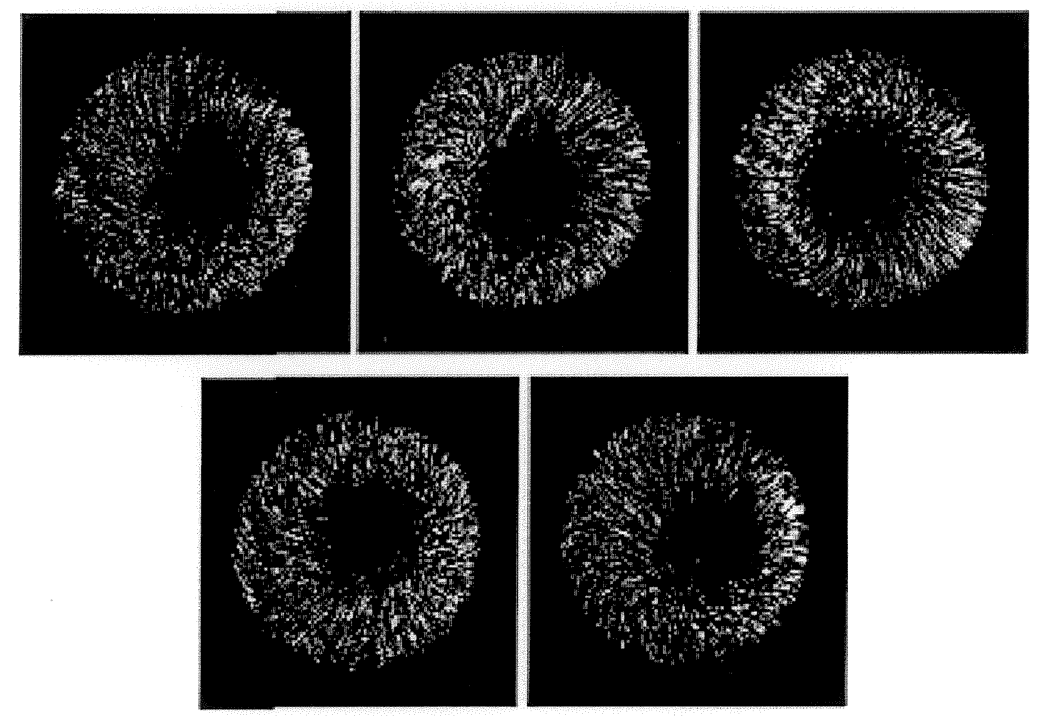

Fig.4. A sequence of flow pattern in the horizontal cross-section in one oscillatory period

Results of the experiments show that the oscillatory mode depends on the aspect ratio as well as the volume ratio of the liquid bridge. Therefore, non-dimensional parameter $d_{\min } / d_{0}$ is introduced where $\mathrm{d}_{\text {min }}$ is the minimum diameter (or the neck-diameter) of the liquid bridge. In experiments of liquid bridges of different volume ratio, we have measured the critical temperature $(\Delta T)_{c}$ on which the oscillatory thermocapillary convection started. The curve of $(\Delta \mathrm{T})_{\mathrm{c}}$ to $\mathrm{V} / \mathrm{V}_{0}$ was composed of two parts that have different oscillatory modes. For example, a liquid bridge of geometrical aspect ratio $A=l / d_{0}=$ $0.6, d_{0}=5 \mathrm{~mm}$ 


\begin{tabular}{ccc}
$\begin{array}{c}\text { Ratio Width } \\
d_{\min } / d_{0}\end{array}$ & $\begin{array}{c}\text { Azimuthal mode } \\
\mathrm{m}\end{array}$ & $\begin{array}{c}\text { Related State } \\
\end{array}$ \\
\hdashline 0.88 & 1 & $\begin{array}{c}\text { oscillatory } \\
\text { steady } \\
\text { oscillatory }\end{array}$ \\
$0.88 \sim 0.90$ & $\ldots$ & 2
\end{tabular}
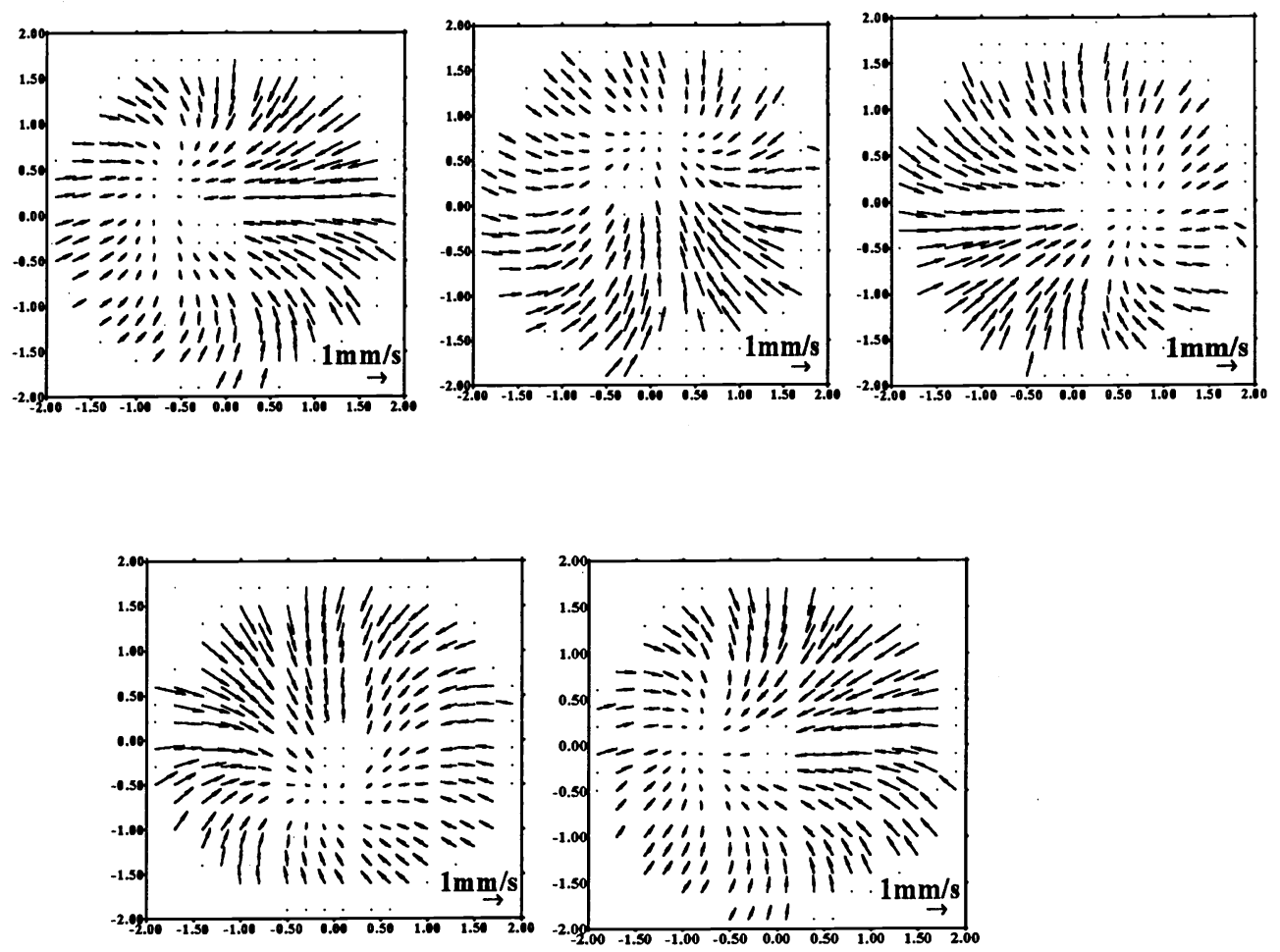

Fig.5. A sequence of velocity field in the horizontal cross-section in one oscillatory period

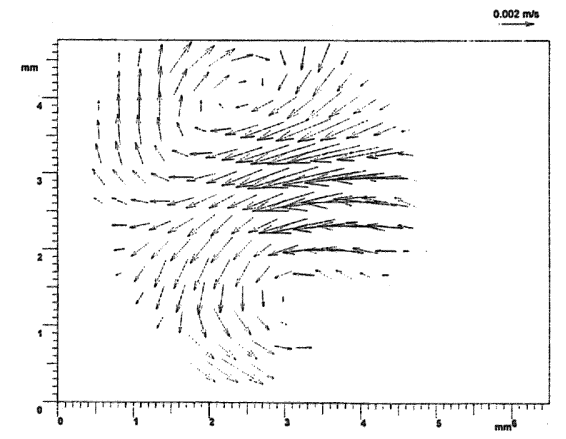

(1) $l / d=0.8$

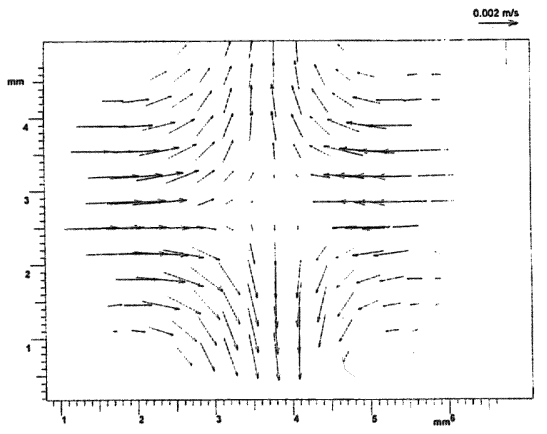

(2) $l / d=0.6$ 


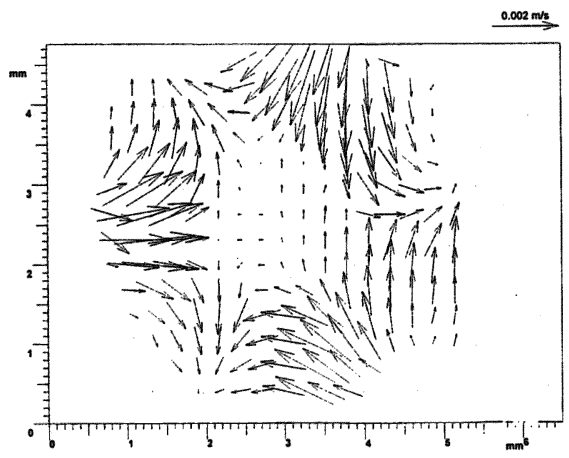

(3) $l / d=0.4$

Fig. 6. The velocity fields in the horizontal cross-section corresponding to different aspect ratio

Fig. 7 give the two-dimensional velocity fields corresponding to different volume ratio of the liquid bridge for the cases of (1) $d_{\min } / d_{0}=0.8$, (2) $d_{\min } / d_{0}=0.89$ and (3) $d_{\min } / d_{0}=0.95$.

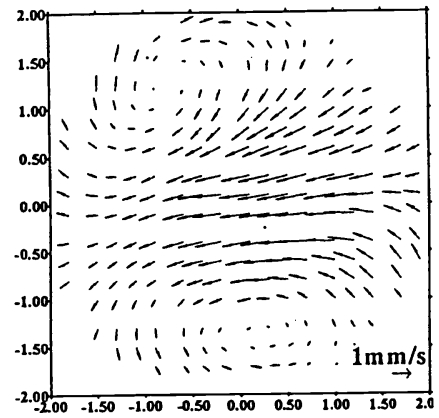

(1) $d_{\min } / d_{0}=0.8$

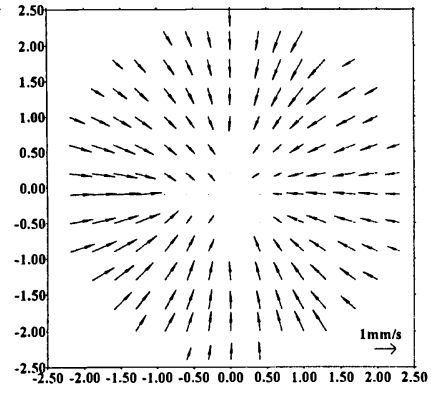

(2) $d_{\text {min }} / d_{0}=0.89$

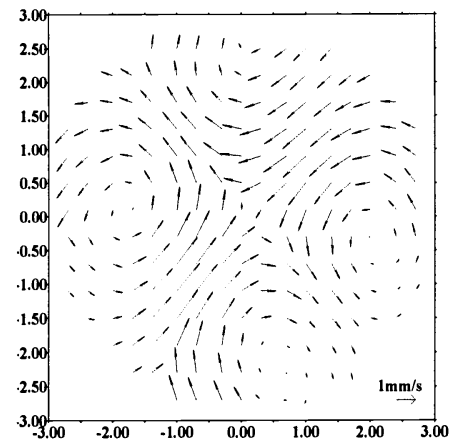

(3) $d_{\text {min }} / d_{0}=0.95$

Fig. 7. The velocity fields in the horizontal cross-section corresponding to different volume ratio

\section{Conclusion}

Using the system of digital particle image velocimetry (DPIV) of DANTEC, we obtained a 2-D flow field of small size in a liquid bridge. Visualization of the oscillatory convection indicates, the oscillatory mode depends on the aspect ratio and volume ratio of the liquid bridge. Our calibration shows that the accuracy of the velocimetry is greater than $5 \%$ in our measurement.

\section{References}

[1] Kamotani Y., Ostrach S., Vargas M. , "Oscillatory thermocapillary convection in a simulated floating-zone configuration", J. Cryst. Growth, Vol. 66, (1984), pp. 83-88.

[2] Chen, Q. S., Hu, W. R., "Numerical investigation on a simulation model of floating zone convection" Int. J.Heat Mass Transfer Vol. 40 (1997), pp 757-765

[3] Flow Map Installation \& User's Guide, DANTEC measurement Technology A/S Denmark, 1996 